\title{
Imager-to-radiometer in-flight cross calibration: RSP radiometric comparison with airborne and satellite sensors
}

\author{
Joel McCorkel ${ }^{1}$, Brian Cairns ${ }^{2}$, and Andrzej Wasilewski ${ }^{2,3}$ \\ ${ }^{1}$ NASA Goddard Space Flight Center, Greenbelt, MD, USA \\ ${ }^{2}$ NASA Goddard Institute for Space Studies, New York, NY, USA \\ ${ }^{3}$ Trinovim LLC, New York, NY, USA \\ Correspondence to: Joel McCorkel (joel.mccorkel@ nasa.gov)
}

Received: 9 June 2015 - Published in Atmos. Meas. Tech. Discuss.: 7 October 2015

Revised: 22 February 2016 - Accepted: 24 February 2016 - Published: 8 March 2016

\begin{abstract}
This work develops a method to compare the radiometric calibration between a radiometer and imagers hosted on aircraft and satellites. The radiometer is the airborne Research Scanning Polarimeter (RSP), which takes multi-angle, photo-polarimetric measurements in several spectral channels. The RSP measurements used in this work were coincident with measurements made by the Airborne Visible/Infrared Imaging Spectrometer (AVIRIS), which was on the same aircraft. These airborne measurements were also coincident with an overpass of the Landsat 8 Operational Land Imager (OLI). First we compare the RSP and OLI radiance measurements to AVIRIS since the spectral response of the multispectral instruments can be used to synthesize a spectrally equivalent signal from the imaging spectrometer data. We then explore a method that uses AVIRIS as a transfer between RSP and OLI to show that radiometric traceability of a satellite-based imager can be used to calibrate a radiometer despite differences in spectral channel sensitivities. This calibration transfer shows agreement within the uncertainty of both the various instruments for most spectral channels.
\end{abstract}

\section{Introduction}

Remote-sensing instruments used for Earth observation are typically characterized over their spectral, spatial, radiometric, polarimetric, and operating sensitivities before; during; and, in the case of airborne sensors, after science measurements have been taken. However, one way to reduce the cost of satellite sensors is to forgo the use of onboard calibra- tion devices to reduce the complexity, mass, and platform requirements. Another way to reduce cost is to be hosted by another payload, but typical requirements for such a partnership prevent orbital maneuvers required by some onboard calibration methods, namely lunar measurements. The NASA Earth Venture - Instrument class missions are intended to have low-to-moderate cost caps and therefore fall within the paradigm of required cost savings that may preclude onboard calibrators and their use due to host spacecraft requirements, or the calibrator cost itself. Cost-saving strategies such as these put science results at risk of being contaminated by calibration issues such as transfer-to-orbit changes and sensor degradation while in operation. To mitigate this risk, vicarious calibration techniques can be used to transfer radiometric traceability from one sensor to another and track degradation over time.

One type of vicarious calibration that does not impose requirements on the sensor or its host spacecraft is cross calibration with other sensors. This is common practice for researchers who need to apply a calibration to their sensor or validate that the current characterization of the sensor is stable. These activities also have the ability to identify systematic biases between sensors (Doelling et al., 2015) and promote discussion of advancing instrument design. For sensors operating in the solar reflective spectrum, most of these studies focus on comparing results of imagers; however, radiometers such as polarimeters can also benefit from these cost-saving techniques by leveraging calibration traceability from other sensors through intercalibration.

In this work we will compare the radiometric calibration of three sensors: Research Scanning Polarimeter (RSP), Air- 
borne Visible/Infrared Imaging Spectrometer (AVIRIS), and Landsat 8 Operational Land Imager (OLI). RSP is an airborne radiometer that scans in the along-track direction and measures the total radiance together with the Stokes parameters $Q$ and $U$ in nine narrow spectral channels spread across the solar reflective spectrum (Cairns et al., 1999). As described by its name, AVIRIS is an airborne imaging spectrometer that operates in the $365-2495 \mathrm{~nm}$ spectral range with spectral channels of approximately $10 \mathrm{~nm}$ bandwidth and $10 \mathrm{~nm}$ sampling. To form an image, AVIRIS scans in the cross-track direction at $12 \mathrm{~Hz}$ over a full field of view of $34^{\circ}$ with 677 spatial samples. RSP and AVIRIS were hosted on the ER-2 aircraft in spring 2014 for the HyspIRI Preparatory Airborne campaign where the instruments took simultaneous measurements. One particular flight line on 31 March 2014 was contemporaneous with an OLI overpass. OLI is a push broom imager with a $185 \mathrm{~km}$ swath width measuring in nine solar reflective spectral channels, of which one is panchromatic across the visible to near infrared. The multispectral channels have $30 \mathrm{~m}$ ground sample distance, and the panchromatic channel has $15 \mathrm{~m}$ sampling but is not used in this work. This work uses the coincident data sets of these three sensors to study methods for characterizing a radiometer using cross calibration to imagers.

Specifically, we want to transfer the calibration from Landsat 8 OLI to AVIRIS and, finally, to RSP. The calibration of OLI is well understood and was carefully characterized prior to launch. It is closely monitored with onboard calibrators and many vicarious techniques (Knight et al., 2014; Czapla-Myers et al., 2015; Morfitt et al., 2015). AVIRIS is also carefully characterized, but resources for airborne instruments and associated calibration and accuracy rarely approach that of a Landsat-class sensor. Therefore, the goal here is to link the radiometric calibration of RSP to OLI using AVIRIS as a transfer. This can be done under the assumption that the AVIRIS calibration accuracy is slowly varying over the spectrum, allowing the calculation of a smooth transfer curve to derive OLI-based radiance from AVIRIS data. This spectral analysis was done to ensure that differences in the spectral sensitivities of the sensors are accounted for properly in the comparisons of their measurements. Both OLI and AVIRIS have higher spatial resolution than RSP, allowing for the proper account of differences in the spatial response of the measurements of each sensor.

\section{Method}

The spectral responses of Landsat 8 OLI and RSP are shown in Fig. 1a. There are several spectral channels where there is some overlap between the two sensors, viz., OLI-2 and RSP2, OLI-3 and RSP-3, OLI-5 and RSP-5, OLI-6 and RSP-7, and OLI-7 and RSP-9. However, the differences in channel centers and bandwidths mean that there are non-negligible differences in measurements of the scene below obtained by
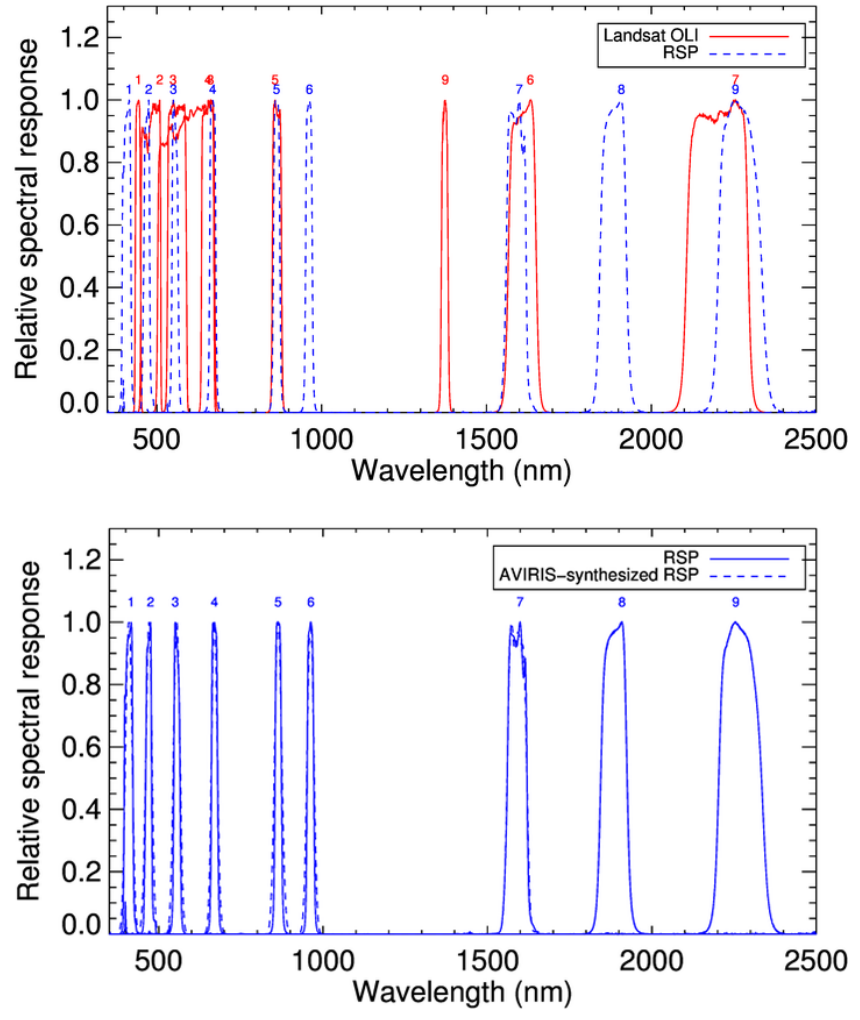

Figure 1. (a) Spectral responses of Landsat 8 OLI as a solid red line and RSP as a dashed blue line. (b) AVIRIS spectral channels are approximately $10 \mathrm{~nm}$ wide and Gaussian-shaped with $10 \mathrm{~nm}$ spacing from 365 to $2495 \mathrm{~nm}$, and weighted sums (dashed lines) have been used to match the RSP spectral channels (solid lines).

the RSP and OLI. Radiometric calibration can still be transferred between OLI and RSP if the scene used for comparison is well understood, either with additional measurements or using a known test site. This work will use spectral knowledge provided by AVIRIS, which was hosted on the ER-2 aircraft at the same time as RSP. The ability to synthesize the RSP spectral channels using a weighted sum of AVIRIS channels is shown in Fig. 1b, where channel centers and bandwidths are matched to better than $1 \%$. Another method to retrieve spectral information is to use desert test sites known as pseudo-invariant calibration sites (PICSs) that are commonly used for intercalibration of space-based sensors operating in the solar reflective spectral region. Many of these PICS are in the Sahara and are measured at least once per day from near-polar orbits. The primary advantage of the PICS methodology is that frequent comparisons provide excellent data for trending and reducing noise in results. However, the PICS methodology largely relies on empirical fits that still need further work to establish robust radiometric traceability. The optimal calibration solution is to use both vicarious methodologies: coincident underflights to establish absolute radiometric calibration and PICS-like methods for radiometric trending. 


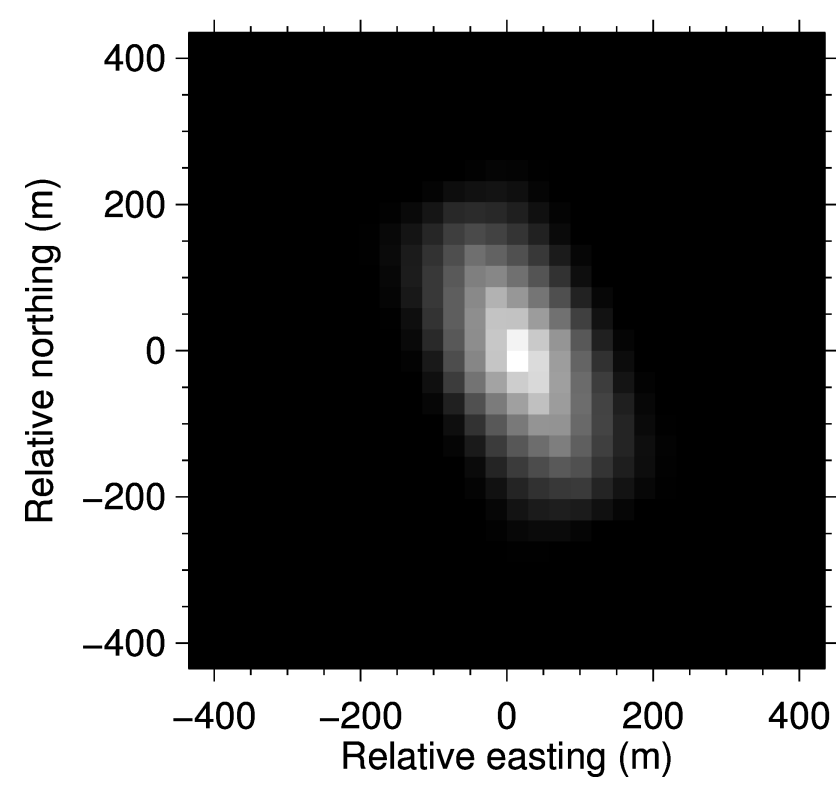

Figure 2. Modeled spatial response of a nadir RSP measurement where the long direction is along track (scan direction).

The sensors studied in this work operate at various spatial resolutions: RSP is a multichannel scanning radiometer that samples in the along-track direction with approximately $220 \mathrm{~m}$ instantaneous field of view for the nadir measurements used in this work; OLI is a push broom imager with a $30 \mathrm{~m}$ ground sample distance; AVIRIS is a spectroradiometer that scans in the cross-track direction to form an image with a $16.1 \mathrm{~m}$ pixel size at nadir. It makes sense to calculate signals from OLI and AVIRIS that are spatially equivalent to RSP, since RSP has the largest footprint of the three sensors. This is done by modeling the spatial response of RSP and convolving it with spatially gridded OLI and AVIRIS data.

The spatial response of RSP is circular with $14 \mathrm{mrad}$ extent and has $14 \mathrm{mrad}$ of drag smear in the scan direction. The resulting spatial response of a measurement is estimated to have a triangular distribution of 28 in the scan direction and $14 \mathrm{mrad}$ in the cross-track direction. The response shape is rotated to match the heading of the aircraft at the time of measurement, and its center is assigned a location according to the associated geolocation information. A spatial response mask, shown in Fig. 2, is calculated at the resolution of the image we are comparing to, either OLI or AVIRIS. This calculation also depends on the distance between the RSP and the ground, which is shown in Fig. 3 for the measurements used in this work.

There are 152 Earth-view samples collected during each RSP scan. The sample closest to nadir-view geometry within each scan is selected for comparison with the other sensors. The geographic location of each RSP measurement, provided in the $\mathrm{L} 1$ data product, is used to find the corresponding measurement within the AVIRIS and OLI data. A $60 \times 60$ pixel

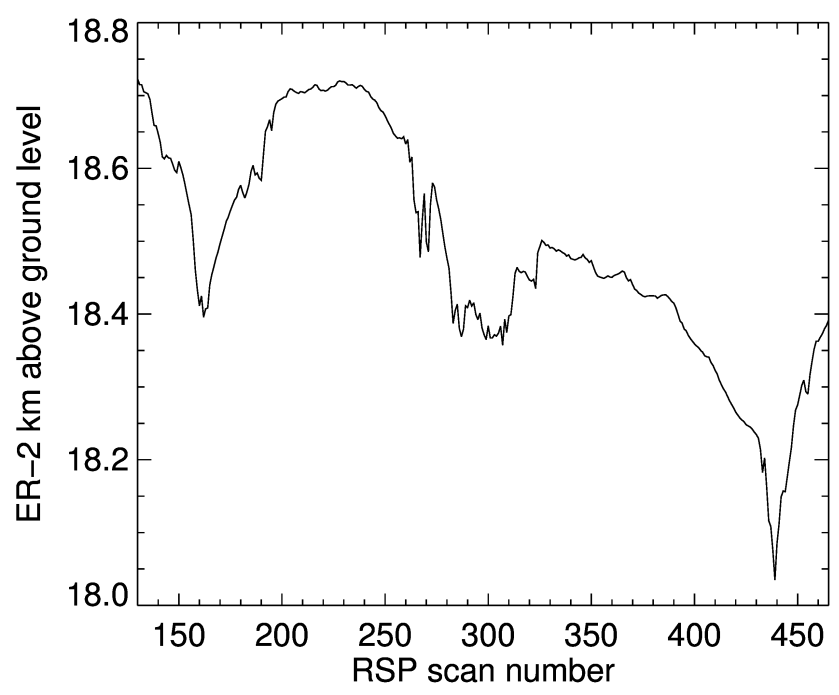

Figure 3. Although the ER-2 holds absolute altitude within tens of meters, the size of the RSP footprint will depend on the sensor's height above ground level, which varies by almost a kilometer in the data set used here.

area centered on the AVIRIS or OLI pixel that best matches the RSP nadir measurement location is extracted from the AVIRIS and OLI imagery for each RSP measurement. The apodization mask that emulates the RSP spatial response is multiplied by each subset extracted from the AVIRIS and OLI imagery. Summing this product and dividing by the sum of the apodization mask provides AVIRIS and OLI signals that have equivalent spatial response to RSP with nearly the same viewing geometry.

Temporal, spectral, and spatial parameters have been discussed and accounted for in our calibration comparison of radiometric response. In Table 1 we provide more detail regarding the uncertainties in the knowledge of offset, radiometric gain, and nonlinearity of response for the RSP. The dark values for the RSP are determined using nine views of a dark reference after a domain controller $(\mathrm{dc})$ restore that resets an integrator. The consequent uncertainties in the determination of this dark level caused by noise are given in digital numbers (DN) and also normalized radiance units in Table 1 in the rows labeled $\sigma_{\text {dark }}$. The RSP also has a pre-dc restore measurement that is used to track drifts in the offset within a scan. The scan period is $0.8409 \mathrm{~s}$, and the amount of drift is currently negligible with a worst case drift of less than $0.05 \mathrm{DN}$ and typical drifts of $0.005 \mathrm{DN}$. The dark reference is located $180^{\circ}$ from the nadir view and should therefore be fairly immune to contamination by scene radiance. However, there is always some leakage/scattering of scene light into the dark reference, and near-field observations of a large aperture integrating sphere provide a good upper bound on such contamination, since the solid angle subtended by the sphere is large. Scattering off blackened and baffled surfaces is largest at the shortest wavelengths, which is why the contamination 
of the dark reference is largest at $410 \mathrm{~nm}$. Even so it is only $0.08 \%$ of the scene radiance, causing negligible radiometric errors. The radiometric uncertainty given in Table 1 has two sources. The first is the transfer uncertainty from the NIST standard source to the secondary standard source that is used for calibration: in this case a sphere (e.g., http://cf.gsfc. nasa.gov/docs/Error/750SlickXferUncert.html). The second source of uncertainty is the variation in the calibration, or gain, coefficient when multiple lamp levels are being fitted. These two sources of uncertainty are considered to be independent, and they are therefore added in quadrature (sum of squares). An additional uncertainty at $1880 \mathrm{~nm}$ is absorption by water vapor in the path through the sphere, which yields an additional uncertainty of $7 \%$ and is the primary source of uncertainty at that wavelength. Repeatability of the determination of the calibration coefficients $(\sim 1 \%$ except for the $1880 \mathrm{~nm}$ band) is compatible with the uncertainties given here and likely changes in instrument performance after multiple ER-2 flights. Nonlinearity in the RSP detector responses can be assessed by fitting an equation of the form $\mathrm{RSP}_{\text {intensity }}=a \times$ Lamp $_{\text {Radiance }}^{b}$ to data from calibrations at multiple radiance levels. Deviations of the fitting parameter, $b$, from unity indicate the level of nonlinearity. The last row in Table 1 shows that nonlinearity in the RSP response is less than $0.5 \%$ in all bands except the $1880 \mathrm{~nm}$ band. Since this band is sensitive to small variations in relative humidity during the course of a calibration, the larger nonlinearity in that case is likely to be caused by environmental variations. Given the uncertainty in the calibrated radiances, it is not possible to reject the hypothesis that the RSP sensor response is linear. The RSP sensor used in this study was radiometrically calibrated at NASA Ames Research Center, looking down into a $30 \mathrm{in}$. spherical integrating sphere at seven different lamp levels (cf. Cairns et al., 1999). In Sect. 3 we show a comparison between RSP and AVIRIS over a wide dynamic range that demonstrates that the primary difference between the sensors is the radiometric gain. The next section presents assessment and results of using data that have been put on a common spatial scale to compare radiometric gain among the sensors.

\section{Results}

RSP, AVIRIS, and OLI data used in this work were downloaded from each sensor's respective server on 1 September 2014, and red-green-blue snapshots from the imagers are shown in Figs. 4 and 5, where the right image in Fig. 5 is a magnification in the OLI image of the area measured by RSP and AVIRIS. The red line in Fig. 4 shows the positions of the nadir RSP measurements, and the associated numbers indicate the scan number for this leg. The red lines in Fig. 5 correspond to the same locations in the OLI imagery.

Ivanpah Playa is seen in the magnified image of Fig. 5 and is the long, narrow, bright feature along the eastern edge of

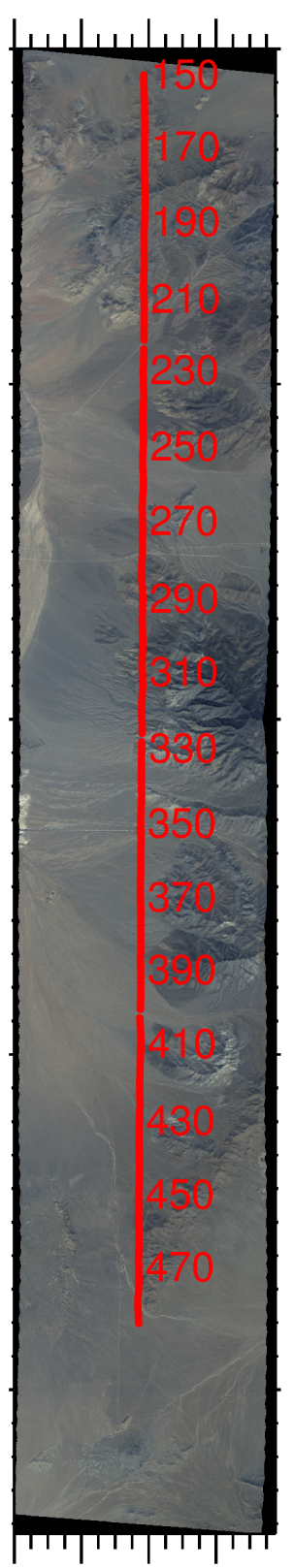

Figure 4. This image shows AVIRIS data acquired 31 March 2014 during 18:13-18:17 UTC in California near the southwestern California-Nevada border. The red line shows the location of the center of nadir RSP measurements, where the numbers indicate the scan number.

the image. Other flight lines flown by the ER-2 on this campaign day measured this commonly used radiometric calibration test site, but not at the time of the Landsat overpass. While there was a research team taking surface reflectance and atmospheric measurements near the center of the playa as part of AVIRIS calibration activities, this work focuses on the intercalibration performance between the RSP and the airborne and satellite imagers. We therefore use coincident measurements of the three sensors: the AVIRIS and RSP data 
Table 1. Uncertainties in dark reference determination, contamination of dark reference by scene radiance, radiometric gain and nonlinearity in response.

\begin{tabular}{lrrrrrrrrr}
\hline Band (nm) & 410 & 470 & 555 & 670 & 865 & 960 & 1590 & 1880 & 2260 \\
$\sigma_{\text {dark (DN) }}$ & 0.22 & 0.22 & 0.22 & 0.17 & 0.23 & 0.20 & 0.27 & 0.28 & 0.27 \\
$\sigma_{\text {dark (norm. rad.) }}$ & $1.5 \mathrm{E}-5$ & $1.2 \mathrm{E}-5$ & $1.1 \mathrm{E}-5$ & $1.0 \mathrm{E}-5$ & $0.8 \mathrm{E}-6$ & $1.0 \mathrm{E}-5$ & $1.0 \mathrm{E}-5$ & $1.0 \mathrm{E}-5$ & $1.0 \mathrm{E}-5$ \\
Dark Cont. (\%) & 0.08 & 0.03 & 0.01 & 0.005 & 0.005 & 0.005 & 0.003 & 0.005 & 0.005 \\
Radiometric & 1.6 & 1.3 & 1.2 & 1.1 & 0.9 & 0.9 & 0.8 & 7.0 & 2.8 \\
Uncertainty (\%) & & & & & & & & & \\
Nonlinearity* (\%) & 0.3 & 0.2 & 0.2 & 0.4 & 0.4 & 0.2 & 0.2 & 0.7 & 0.5 \\
\hline
\end{tabular}

* Notes: the natural log of the fitting parameter, $b$, described in the text multiplied by 100.
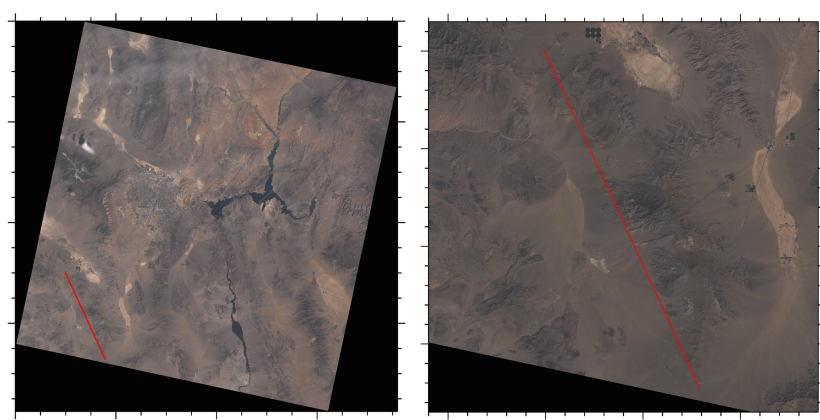

Figure 5. Landsat 8 OLI scene of southern Nevada captured on 31 March 2014 at 18:16 UTC is shown on the left. The ER-2 flight path and associated RSP and AVIRIS measurements were collected along the red line during 18:13-18:17 UTC on the same day. The flight path is $24.7^{\circ}$ west of north with total path length of $57.1 \mathrm{~km}$. The right image is a magnified view of the area of the flight line.

used here were collected during 18:13-18:17 UTC, and the Landsat overpass was at 18:16 UTC on 31 March 2014.

There are 336 discrete measurement locations along the red line that is overlaid on Figs. 4 and 5. As described in the previous section, the modeled spatial response of RSP is convolved with the AVIRIS and OLI imagery to obtain signals that are spatially equivalent to the RSP spatial response. These calculated signals are shown in Fig. 6 for the near-infrared channel of the sensors: OLI channel 5 and RSP channel 5. The spectral response data of RSP channel 5 was used to band-average the AVIRIS signal for this plot. The agreement in shape and fluctuations in these lines (correlation of 0.998) indicates high-quality geolocation of the sensors as well as similar calibration performance. Another measure of agreement between the sensors is the similarity of the variability in each sample as shown in Fig. 7, although only AVIRIS and OLI can be gauged in this manner since each RSP measurement is a single nadir value. Other spectral channel pairs show similar agreement in shape and fluctuations, but the curves have offsets from each other. One reason for these biases is differences in spectral channel location and shape that mean the sensors are measuring different spectral regions. Another culprit is radiometric calibration disagreement between the sensors.

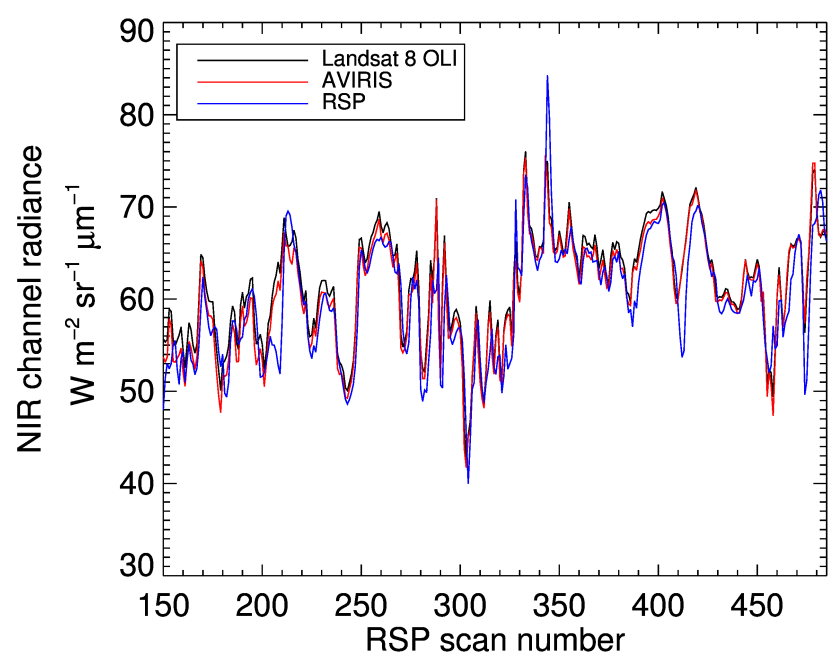

Figure 6. The signal of OLI, AVIRIS, and RSP along the line formed by locations of RSP nadir measurements. The OLI and AVIRIS imagery was spatially averaged to match the RSP footprint. The AVIRIS spectrum was band-averaged to match RSP channel 5.

One way to compare the radiometric signals from these sensors and alleviate spectral response differences is to compare the multispectral sensors, RSP and OLI, to the imaging spectrometer. The spectral response of each spectral channel of the imagers is used to calculate the RSP- and OLIspectrally equivalent signals of AVIRIS data. This means that AVIRIS becomes the basis for radiometric comparison, and results of these calculations are shown in Fig. 8. The vertical axis is in units of percent difference from AVIRIS-based radiance, so circles on the zero line would represent perfect agreement, and negative values occur when AVIRIS reports a lower radiance than the sensor it is being compared to. All points are within $5 \%$ agreement except for channel 1 of both sensors and the channels affected by water vapor absorption. Causes for difference in radiance are primarily due to disagreements in sensor characterization, but factors such as small geolocation errors and atmospheric differences can be additional sources of uncertainty in this comparison. However, geolocation errors are minimized by only selecting measurements that have $3 \%$ variability or less. This screen- 


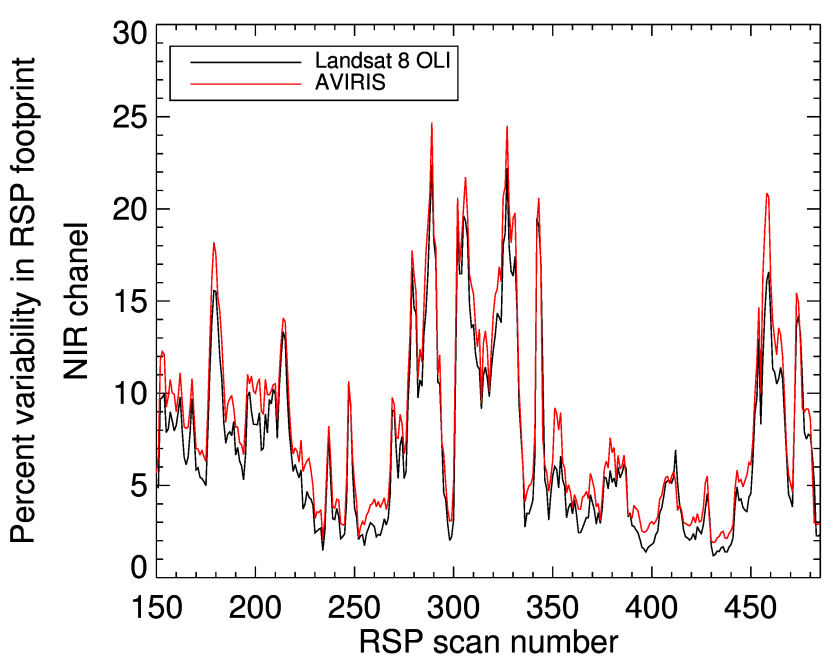

Figure 7. The percent standard deviation of each measurement along the flight path. There about 100 AVIRIS and OLI pixels within each RSP measurement footprint, and the variability of these pixels provides an assessment of the land surface uniformity within the RSP footprint. Measurements with high variability will be more sensitive to geolocation errors among the sensors and can be screened from further processing.

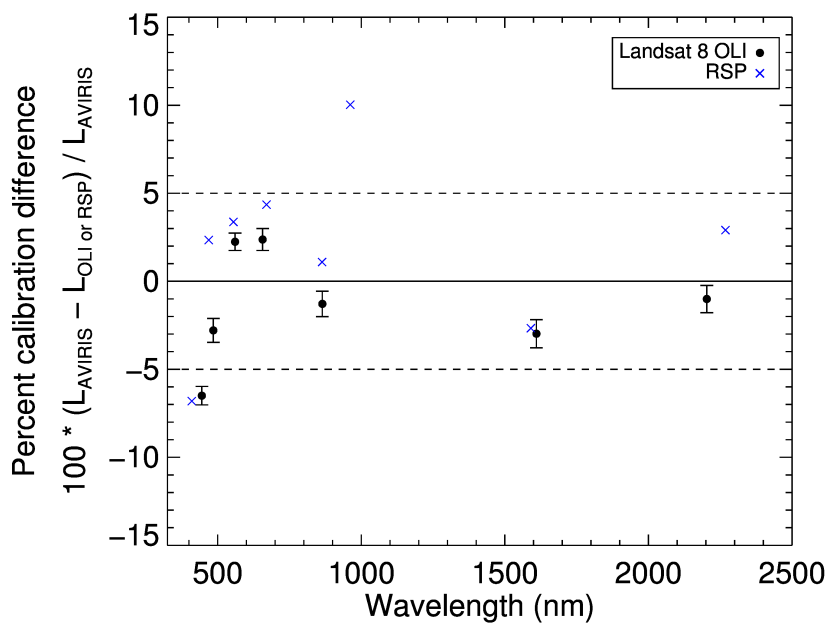

Figure 8. The percent difference in radiance between bandaveraged AVIRIS and the multispectral sensors, RSP and OLI.

ing process, which selected 64 of the 336 samples, selects only samples over uniform areas of surface cover, which reduces contamination of results caused by potential geolocation mismatch between the sensors, and atmospheric differences are minimized by the use of coincident data sets.

While agreement of RSP and OLI with AVIRIS is quite good, with only one window channel showing a difference of greater than $5 \%$, we want to derive a more direct intercalibration link between the RSP and OLI calibration to demonstrate a method for assessing consistency between a radiometer and an orbiting multispectral imager. One difficult aspect

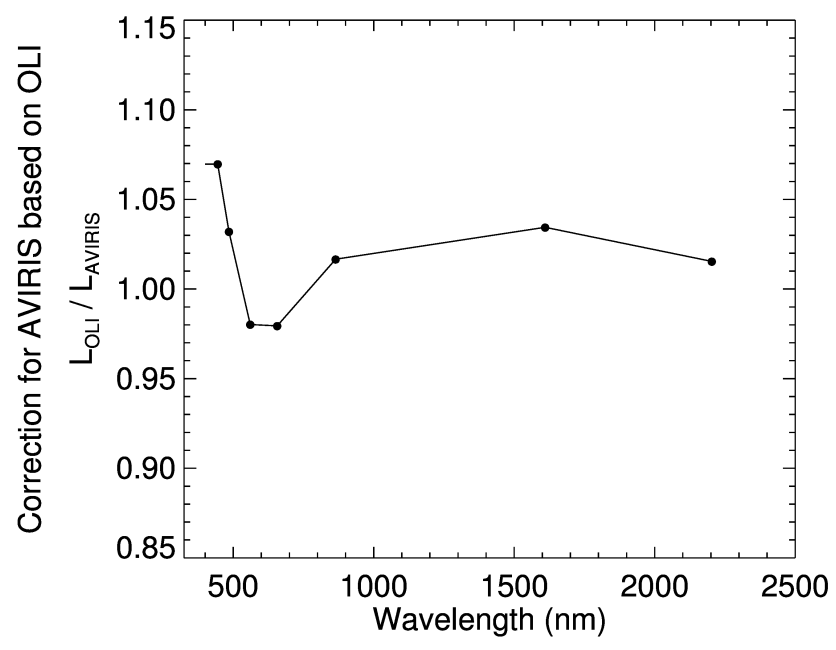

Figure 9. The ratio difference in spectral radiance between OLI and AVIRIS is used to calculate a correction curve. The multispectral points (circles) are interpolated to the spectral location of each AVIRIS channel (shown as line).

of this problem is that the spectral channels of the radiometer, RSP, and the imager, OLI, do not have identical spectral sensitivities. There are two general methods to solve this problem: model the spectral variability of the test site or measure it. The first has been done in other work by using in situ measurements (Teillet et al., 2001; McCorkel et al., 2013). The second is possible if we use an imaging spectrometer as a transfer radiometer, such that the calibration knowledge of OLI is applied to AVIRIS and subsequently to RSP. Except for the spectral channels affected by water vapor, the data of both sensors in Fig. 8 follow a similarly shaped curve, suggesting that RSP and OLI calibration behaviors are in closer agreement with one another than with AVIRIS. The curve could be used to create a correction to convert AVIRIS radiances to have an OLI-equivalent radiance scale. Such a curve is shown as a ratio in Fig. 9 such that multiplying AVIRISmeasured spectral radiance by this factor will provide a signal with radiometric traceability to OLI.

Creating a calibration curve for an imaging spectrometer based on a multispectral signal may seem counterintuitive, but the prevalence of multispectral imagers with wellunderstood calibration currently on orbit means that such a method has a wide range of application. There is concern that, since the multispectral sensors have zero information between their channels, they can not provide calibration information to the imaging spectrometer. However, like many things in nature, variations in imaging spectrometer response are typically spectrally continuous rather than displaying discrete, step-function-like changes. This is due to the spectrally continuous nature of the characteristics of the optical components used in the instrument such as mirrors, diffraction gratings, and detectors. These components have reflectivities, efficiencies, and responsivities that vary slowly with wave- 


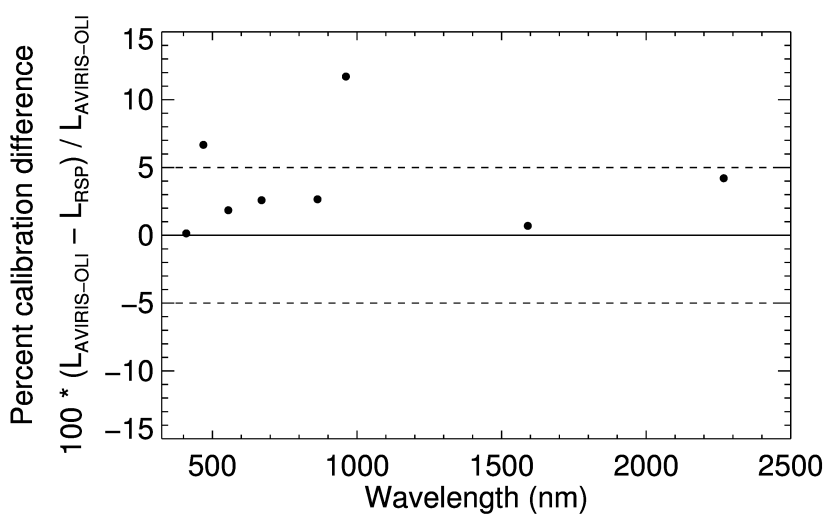

Figure 10. Percent difference between RSP and OLI-calibrated AVIRIS.

length over the spectral regions they were designed to operate over. Even an imaging spectrometer like AVIRIS that uses four separate spectrometers to cover the full solar reflective spectral range will show continuous behavior since discontinuities are necessarily minimized as part of the laboratory calibration process.

The OLI-calibrated AVIRIS spectral radiance is bandaveraged with the RSP spectral response and compared with RSP-measured spectral radiance. The difference between these signals is shown in Fig. 10. Agreement is within 5\% except for the 470, 960, and $1880 \mathrm{~nm}$ RSP channels. The $1880 \mathrm{~nm}$ data point is off the scale in Fig. 10. The 960 and $1880 \mathrm{~nm}$ spectral bands are strongly affected by water vapor absorption, adding difficulty to characterizing absolute radiometric response, even in controlled laboratory conditions. The cause of disagreement of RSP channel $2(470 \mathrm{~nm})$ is unclear but is possibly an artifact of the rapidly changing OLI-to-AVIRIS calibration curve in this spectral region. Two examples of comparisons between RSP and AVIRIS over a wide dynamic range are given in Fig. 11. When the ratio of the spectral radiances of the two sensors is plotted against the reciprocal of one of the sensors' spectral radiances, any influences of nonlinearity or offset differences are emphasized. If radiometric gain is the only difference, the data should fall on a horizontal line, while if there is an offset difference the data will have a finite linear slope. In Fig. 11a the data clearly show the difference in radiometric gain between AVIRIS and RSP at $410 \mathrm{~nm}$ that was noted previously. In Fig. 11b there is clearly a linear trend that suggests a difference in offsets between the two sensors for the $960 \mathrm{~nm}$ band. However, since the dynamic range in the signals includes variations in water vapor for this band that is used for water vapor retrievals and the spectral responses of the synthetic RSP band and the actual RSP band do not match perfectly, the difference in offsets may be caused by differing in spectral responses.
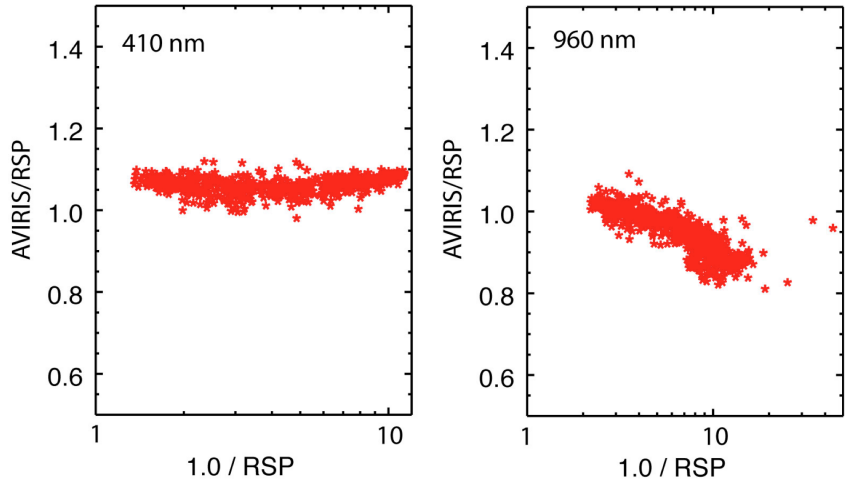

Figure 11. Comparisons of RSP and AVIRIS over a wide dynamic range for best $(410 \mathrm{~nm})$ and worst $(960 \mathrm{~nm})$ cases.

\section{Conclusions}

In this paper we compared the radiometric calibration of an airborne radiometer (RSP) to that of an airborne imaging spectrometer (AVIRIS) and a satellite imager (Landsat 8 OLI). We modeled the spatial response of RSP to calculate spatially equivalent signals of the imagers for this cross calibration. First we compared band-averaged AVIRIS radiance signals to the multispectral sensors, RSP and OLI, by using each sensor's spectral response. Most spectral channels of OLI and RSP agreed with AVIRIS to within the combined sensor uncertainties. However, this comparison does not directly compare RSP to OLI, and it is not straightforward to do so since these sensors have different spectral sensitivities.

Next, we investigated a method to transfer the calibration of OLI to RSP using AVIRIS as a transfer. The AVIRIS signal was adjusted to match the OLI radiometric calibration using a correction factor found by comparing OLI and AVIRIS signals. The OLI-based AVIRIS continuous-spectrum radiance signal was then band-averaged to RSP's spectral channels for comparison with the RSP-measured signal. Except for the $470 \mathrm{~nm}$ channel and channels affected by water vapor, the results were consistent with a $\pm 2.5 \%$ spread in the radiometric calibration of RSP and OLI. This is in contrast to the spread of the RSP and OLI comparison to AVIRIS mentioned above, which was $\pm 5 \%$. This suggests that RSP and OLI are in good agreement except for a possible small, $2.5 \%$, bias.

Future efforts will work to close the radiometric link between RSP and OLI presented here. This is possible with laboratory experiments to compare the sensor calibration of RSP and the Landsat Transfer Radiometer (LXR) using the same extended source. This would be a valuable comparison since the LXR was used during prelaunch laboratory testing of OLI to provide radiometric traceability to national standards - the LXR would therefore be a laboratory surrogate for OLI (Markham et al., 1998; Butler and Barnes, 2003). Analysis of these data will use much of the same spectral 
analysis presented here, and results will be directly comparable to those presented above.

The methodology developed here is scalable to a satellitebased radiometer, such as the Aerosol Polarimetry Sensor (APS) had it successfully launched. Also, since an APSlike radiometer will have a much larger terrestrial footprint, more imagers are available for cross calibration due to relaxed spatial-resolution requirements. For example the 250$1000 \mathrm{~m}$ spatial sampling of MODIS and VIIRS can be used to emulate the spatial response of an APS-like radiometer, while the cross-track swath of AVIRIS $(11 \mathrm{~km})$ is sufficient to allow for the use of AVIRIS in conjunction with such imagers. Advantages of sensors like these in addition to Landsat imagers for cross calibration include frequent revisit period and accurate radiometric calibration (Xiong and Barnes, 2006; Xiong et al., 2014).

As science questions grow and the availability of science funding becomes more limited, instrument concepts need to be developed that provide the most benefit for the least amount of cost and risk. One way to reduce cost of building an instrument is to forgo onboard calibrators and costly testing associated with them, and rely on cross-calibration methods, such as the one developed in this work, to use the well-understood calibration of flagship sensors.

Acknowledgements. We would like to thank the NASA Radiation Sciences and Ocean Biology and Biogeochemistry programs and the HySPIRI airborne preparatory program for funding the acquisition of airborne data that is used in this paper. We would also like to thank the NASA Climate Absolute Radiance and Refractivity Observatory (CLARREO) Decadal Survey mission for support in advancing sensor intercalibration techniques shown in this work.

Edited by: H. Worden

\section{References}

Butler, J. J. and Barnes, R. A.: The use of transfer radiometers in validating the visible to shortwave infrared calibrations of radiance sources used by instruments in NASA's Earth Observing System, Metrologia, 40, S70-S77, 2003.

Cairns, B., Russell, E. E., and Travis, L. D.: Research scanning polarimeter: calibration and ground-based measurements, in: SPIE's International Symposium on Optical Science, Engineering, and Instrumentation, International Society for Optics and Photonics, 186-196, 1999.
Czapla-Myers, J., McCorkel, J., Anderson, N., Thome, K., Biggar, S., Helder, D., Aaron, D., Leigh, L., and Mishra, N.: The groundbased absolute radiometric calibration of Landsat 8 OLI, Remote Sens., 7, 600-626, 2015.

Doelling, D. R., Wu, A., Xiong, X., Scarino, B. R., Bhatt, R., Haney, C. O., Morstad, D., and Gopalan, A.: The Radiometric Stability and Scaling of Collection 6 Terra-and Aqua-MODIS VIS, NIR, and SWIR Spectral Bands, IEEE Geosci. Remote Sens. Soc., 53, 4520-4535, 2015.

Knight, E. J. and Kvaran, G.: Landsat-8 operational land imager design, characterization and performance, Remote Sens., 6, 1028610305, 2014.

Markham, B. L., Schafer, J. S., Wood Jr., F. M., Dabney, P. W., and Barker, J. L.: Monitoring large-aperture spherical integrating sources with a portable radiometer during satellite instrument calibration, Metrologia, 35, 643-648, 1998.

McCorkel, J., Thome, K., and Lockwood, R. B.: Absolute radiometric calibration of narrow-swath imaging sensors with reference to non-coincident wide-swath sensors, IEEE Geosci. Remote Sens. Soc., 51, 1309-1318, 2013.

Morfitt, R., Barsi, J., Levy, R., Markham, B., Micijevic, E., Ong, L., Scaramuzza, P., and Vanderwerff, K.: Landsat-8 Operational Land Imager (OLI) radiometric performance on-orbit, Remote Sens., 7, 2208-2237, 2015.

Teillet, P. M., Fedosejevs, G., Gauthier, R. P., O’Neill, N. T., Thome, K. J., Biggar, S. F., Ripley, H., and Meygret, A.: A generalized approach to the vicarious calibration of multiple Earth observation sensors using hyperspectral data, Remote Sens. Environ., 77, 304-327, 2001.

Xiong, X. and Barnes, W.: An overview of MODIS radiometric calibration and characterization, Adv. Atmos. Sci., 23, 69-79, 2006.

Xiong, X., Butler, J., Chiang, K., Efremova, B., Fulbright, J., Lei, N., McIntire, J., Oudrari, H., Sun, J., Wang, Z., and Wu, A.: VIIRS on-orbit calibration methodology and performance, J. Geophys. Res.-Atmos., 119, 5065-5078, 2014. 